\title{
Morphology of first seven larval stages of the striped soldier shrimp, Plesionika edwardsii (Brandt, 1851) (Crustacea: Decapoda: Pandalidae) from laboratory reared material
}

\author{
JOSÉ MARÍA LANDEIRA ${ }^{1}$, FERNANDO LOZANO-SOLDEVILLA ${ }^{1} \&$ \\ JUAN IGNACIO GONZÁLEZ-GORDILLO ${ }^{2}$ \\ ${ }^{\text {I}}$ Departamento de Biología Animal (UDI Ciencias Marinas), Facultad de Biología, Universidad de La Laguna, Campus Anchieta, \\ 38206, La Laguna, Spain.E-mail: jmlandei@ull.es \\ ${ }^{2}$ Centro Andaluz de Ciencia y Tecnología Marinas, Universidad de Cádiz, c/ República Saharaui s/n, E-11510 Puerto Real (Cádiz), \\ Spain
}

\begin{abstract}
With the idea of starting a research program for the biological study of Plesionika edwardsii, eight ovigerous females were collected with artisanal bottom traps at $220 \mathrm{~m}$ depth in the Southwest of Gran Canaria island, north-western Africa $\left(27^{\circ} 43.864^{\prime} \mathrm{N} 15^{\circ} 47.822^{\prime} \mathrm{W}\right)$, for laboratory larval cultures. The first seven zoeal stages of Plesionika edwardsii were obtained for 20 days after hatching at $23.4 \pm 0.4^{\circ} \mathrm{C}, 10^{\circ} \mathrm{C}$ above adult habitat temperature. The zoeal stages are described and illustrated, constituting the first detailed larval description of the genus Plesionika. The larval morphology is compared with previous larval descriptions attributed to Plesionika spp. collected in plankton tows and with descriptions of the first stage of development of Plesionika acanthonotus reared in laboratory.
\end{abstract}

Key words: Plesionika edwardsii, Pandalidae, larval development, zoea

\section{Introduction}

The genus Plesionika Bate, 1888 is the most speciose genus within the caridean family Pandalidae Haworth, 1825, being represented by more than 80 species (Chan \& Yu 2000). Of these, 12 species have been reported from the north-eastern Atlantic waters (d'Udekem d'Acoz 1999): Plesionika acanthonotus (Smith, 1882), $P$. antigai Zariquiey Álvarez, 1955, P. edwardsii (Brandt, 1851), P. ensis (Milne-Edwards, 1881), P. geniculatus (Milne-Edwards, 1883), P. gigliolii (Senna, 1902), P. heterocarpus (Costa, 1871), P. holthuisi Crosnier \& Forest, 1968, P. martia (Milne-Edwards, 1883), P. narval (Fabricius, 1787), P. rossignoli Crosnier \& Forest, 1968, and P. williamsi Forest, 1964. All of these species have been included in the FAO catalogue of species of interest to fisheries (Holthuis 1980).

The striped soldier shrimp, Plesionika edwardsii, occurs at Atlantic and Indo-Pacific low latitudes. In the eastern Atlantic Ocean, it has been collected in the Ibero-Morrocan region (western Sahara) from Mauritania to Angola, in the Azores, Madeira and Canary Islands archipelagos (Udekem D'Acoz 1999; González et al. 2001) and in the Mediterranean Sea. P. edwardsii is a bathyal species (54 to $649 \mathrm{~m}$ depth) that inhabit mud, sandy and rocky bottoms (González et al. 2001). Although it broods continuously throughout the year, the highest reproductive activity of $P$. edwardsii occurs from April to July (Colloca 2002). This species is frequently caught in trawlers and bottom shrimp-traps in Tunisia, Algeria, Italy, Mediterranean coast of Spain and Canary Islands (Zariquiey Álvarez 1968; Holthuis 1980; Santana et al. 1997; Vafidis et al. 2005). The Spanish fishing fleet has an annual catch between 80-100 tons (González et al. 1992). Moreover, a small artesian fleet has been developed specifically for this fishery in the Canary Islands and Madeira archipelagos in the last decades (Santana et al. 1997). 
The complete larval development of Plesionika species is still unknown. Up to now, the unique reliable and available description obtained from laboratory cultures belongs to the first zoeal stage of Plesionika acanthonotus (Bourdillon-Casanova, 1960) because the Mura \& Pessani's Plesionika edwardsii description is based on a prezoea larvae, poorly documented and does not provide any interesting morphological features (Mura \& Pessani 1994). Several others larvae collected in planktonic samples have been also assigned to the genus Plesionika: Icotopus arcurostris (advanced zoeal stage) (Bate 1888); Pandalidae (Icotopus) species 3 (zoeae IV-IX) (Gurney 1924); Plesionika sp.? (zoeae I-XI) (Kurian 1956); Plesionika sp. (zoea IV) (Bourdillon-Casanova 1960); Plesionika martia? (included in an identification key) (Williamson 1967a); Icotopus sp. (Williamson 1967b); Plesionika sp. (zoeae I-II) (Seridji 1971); Plesionika sp. (zoeae I-XI) (Barnich 1996); Plesionika sp. (zoea I) and Plesionika FSL12 (zoeae I-VII) (dos Santos 1999). (see González-Gordillo et al 2001, for details).

The present study describes the morphological features of Plesionika edwardsii larvae reared in laboratory, in what represents the first detailed larval description of the genus Plesionika. This description represents a useful tool for the identification of $P$. edwardsii from plankton samples and, helps to understand the life history of this commercial species for stock assessment and management.

\section{Material and methods}

Eight ovigerous shrimps were caught in November 2004, during the PANDCAN4 survey, with artisanal bottom shrimp traps at $220 \mathrm{~m}$ depth in Mogán, south-western Gran Canaria (27 $\left.43.864^{\prime} \mathrm{N}-15^{\circ} 47.822^{\prime} \mathrm{W}\right)$. Initially, the specimens were placed in a 1001 aquarium with filtered natural seawater in the laboratory. After $24 \mathrm{~h}$, three specimens with egg mass that were more developed at Stage III (see Thessalou- Legaki 1992) were transferred to individual 21 aquariums. Larval hatching occurred within $72 \mathrm{~h}$, from only one female (the rest died during the culture) and immediately some zoea I larvae were collected for description purposes. Rearing procedure was based on Calado et al. (2003). Actively swimming larvae were transferred to three 121 grey cylindrico-conical fibreglass tanks, at a density of 10 larvae $1^{-1}$, with aerated seawater at a constant temperature of $23.4 \pm 0.4^{\circ} \mathrm{C}$ and subjected to a natural light-dark regime (11:13 L:D ratio). During first and second instars, larvae were fed on Brachionus plicatilis grown with culture Selco ${ }^{\mathrm{TM}}$ at a density of 20 rotifers $\mathrm{ml}^{-1}$. The third instar was fed on a mixture of rotifers $\left(10\right.$ rotifers $\left.\mathrm{ml}^{-1}\right)$ and fresh Artemia sp. nauplii (10 nauplii $\mathrm{ml}^{-1}$ ). The following instars were fed on Artemia sp. metanauplii enriched with DHA Selco ${ }^{\mathrm{TM}}$ at a density of 12 metanauplii $\mathrm{ml}^{-1}$. The tanks were checked daily in order to remove the exuvia and dead larvae; afterwards, the water was changed and food was supplied. Specimens at all zoeal instars were sorted and preserved in $80 \%$ ethanol.

For easier observation of larval structures under microscope, a digestion-stain procedure was carried out. Firstly, entire specimens were placed in a watch glass with $2 \mathrm{ml}$ of heated lactic acid (at approximately $95^{\circ} \mathrm{C}$ ), for about one or two minutes. During this time the preparation was continuously checked under a stereomicroscope to check that tissues digestion was going correctly. This is a critical step of the procedure since specimen handling must be done very carefully in order to avoid setae removal. Immediately after, three drops of Clorazol Black stain ( $0.4 \mathrm{~g}$ Clorazol Black powder dissolved in $75 \mathrm{ml} 70 \% \mathrm{EtOH})$ were added to the heated solution. One minute later, the specimen was removed from the solution and placed on a slide with lactic acid (at room temperature), in order to proceed with the dissection of the mouthparts. Clorazol Black can penetrate the chitin, which facilitates the examination of external morphological features. However, if the specimen is kept for too long in the stain solution the chitin turns very dark and many structures will be very difficult to see. When undesired portions of the tissue matrix were not removed, the dissolving procedure was repeated using heated lactic acid again. At least, 10 specimens of each stage were used to describe the morphology. The seventh stage was described with only 3 larvae because the culture ended for getting material for the morphological analyses. 
The appendages were dissected and mounted in lactic acid and drawn using an interference phase microscope with camera lucida. The preparations were not permanent. Setal counts and other morphological features were performed after Clark et al. (1998). Given the profusion of names assigned to the multiple types of setae shown by decapod larvae, we used the most uniformly encountered setal types in P. edwardsii larvae, according to Pohle \& Telford (1981), Felgenhauer (1992), Ingle (1992), and Garm (2004). The long plumose setae and aesthetascs on distal exopod segments are drawn truncated and setules from setae were omitted from drawings when necessary.

Cephalotorax length (CL) was measured from the tip of the rostrum to the posterior cephalotorax margin. The sizes are given as the arithmetic mean $\pm 95 \%$ confidence intervals. Rostrum length/ frontal lobe length ratio (RS/FL) was done to show the length decreasing of the rostrum through the larval development. The ratio of the $6^{\circ} / 5^{\circ}$ abdominal somites lengths were constant from third stage on and it could be useful to distinguish P. edwardsii from other Plesionika species in the future.

\section{Results}

The first seven zoeal stages of Plesionika edwardsii were obtained for the first 20 days after hatching at $23.4 \pm$ $0.4^{\circ} \mathrm{C}, 10^{\circ} \mathrm{C}$ above adult habitat temperature. The larval morphology of the first stage (zoea I) is described in detail and major features of each larval stage are shown separately. Features of each larval stage as well as changes in appendage setation and setal types are summarized and are given in Table 1. Table 2 provides a detailed list of the characters defining each larval stage, which allows a direct recognition of each stage.

\section{Plesionika edwardsii (Brandt, 1851)}

(Figures 1-7)

\section{First zoea}

Cephalotorax (Figures 1A,D). Flattened. Anterior ventral margin with a pterygostomian spine and with two anteroventral spines. Eyes sessile. Dorsomedian tubercle on anterior and posterior part of cephalotorax. Rostrum slender and pointed, reaching extremity of antennular peduncle.

Antennule (Figure 2A). Slender with unsegmented peduncle. Exopod bears terminally 1 plumose seta, 1 spatulate seta and 3 aesthetascs. Endopod as a long plumose seta.

Antenna (Figure 3A). Peduncle with a seta near the base of the endopod. Endopod unsegmented, elongated with two terminal setae (plumose and simple). Exopod broad and distally 5-segmented with 11 marginal plumose setae and 1 simple seta on apex.

Mandible. Asymmetrical, consisting of incisor and molar processes, without palp.

Maxillule (Figure 4A). Coxal endite with 5 denticulate setae and 2 plumose setae and microtrichia; basial endite with 4 plumodenticulate cuspidate setae, 1 simple seta and microtrichia; endopod unsegmented with 5 setae forming three groups. Exopod present as a long plumose setae.

Maxilla (Figure 4C). Coxal endite bilobed with 13 setae; basial endites bilobed with 8 setae; endopod unsegmented and tetralobed with 9 setae $(3+2+1+3)$ and microtrichia; exopod with 5 long plumose setae and microtrichia.

First maxilliped (Figure 4D). Coxal endite with 4 plumose setae; basial endite with 12 setae forming 5 groups; endopod 4-segmented with 3, 1, 2, 1+3 setae and not extending beyond middle of exopod. Exopod unsegmented bearing 1 subterminal and 3 terminal plumose natatory setae.

Second maxilliped (Figure 5A). Coxal endite with 1 seta; basial endite with 9 sparsely plumose setae displaying four groups $(1+2+3+3)$; endopod 4 -segmented with 3,1,2 sparsely plumose setae and 5 setae terminally ( 1 simple and 4 serrulate setae); exopod unsegmented with 2 subterminal and 3 terminal plumose natatory setae. 
TABLE 1. Morphological and meristics features of zoeal stages of Plesionika edwardsii. Setal groups on successive segments are separated by a comma and groups of setae on the same segment, or on different lobes of the same endite, are separated by a plus sign (+). RS, rostral spine; FL, frontal lobe; $6^{\circ}$ and $5^{\circ}$, length of $6^{\circ}$ and $5^{\circ}$ abdominal somites respectively; (-), absent. a, aesthetasc; c, spiniform seta; d, plumodenticulate seta; e, sparsely plumose seta; f, plumoserrulate seta; $h$, sparsely hardy plumose seta; $j$, spiny projection; $L$, laterally plumose seta; $m$, microtrichia; $n$, plumose natatory seta; $p$, plumose seta; $r$, serrulate seta; $s$, simple seta; $t$, plumodenticulate cuspidate seta; $v$, spatulate seta.

\begin{tabular}{lcccc}
\hline \multicolumn{1}{c}{ Features } & Z I & Z II & Z III & Z IV \\
\hline $\begin{array}{l}\text { Duration of stage (days) } \\
\text { Cephalotorax }\end{array}$ & 4 & 6 & 7 & 6 \\
Length (mm) & $0.933 \pm 0.021$ & $0.996 \pm 0.030$ & $0.999 \pm 0.027$ & $0.998 \pm 0.034$ \\
Eyes & sessile & stalked funnel-shaped & stalked funnel-shaped & stalked funnel-shape \\
Ratio RS/FL & 4 & 3 & $1.5-2$ & $1.5-2$ \\
Antennule & & & $1 \mathrm{p}, 5 \mathrm{p}, 4 \mathrm{p}+6 \mathrm{p}$ & $1 \mathrm{c}+3 \mathrm{p}, 5 \mathrm{p}, 4 \mathrm{p}+6 \mathrm{p}$ \\
$\quad \begin{array}{c}\text { Peduncle } \\
\text { Endopod }\end{array}$ & long plumose seta & long plumose seta & long plumose seta & long plumose seta \\
Exopod & $3 \mathrm{a}+1 \mathrm{p}+1 \mathrm{v}$ & $3 \mathrm{a}+1 \mathrm{p}+1 \mathrm{v}+2 \mathrm{~s}$ & $4 \mathrm{a}+2 \mathrm{p}+1 \mathrm{~s}$ & $4 \mathrm{a}+2 \mathrm{p}$
\end{tabular}

\section{Antenna}

Peduncle

Endopod

Ratio end/exo

Exopod

\section{Maxillule}

Coxal endite

Basial endite

$2 \mathrm{p}+5 \mathrm{~d}$

$1 c$

$1 \mathrm{p}+1 \mathrm{c}$

$1 c$

$1 \mathrm{p}+1 \mathrm{c}$

$1 \mathrm{c}$

$1 \mathrm{c}$

0

0

$1 / 2$

$1 / 2$

$1 / 2$

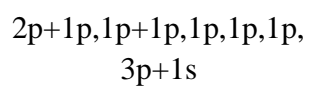

$2 p+1 p, 1 p+1 p, 1 p, 1 p, 1 p, 3$ $\mathrm{p}+1 \mathrm{~s}$

$5 p+2 p, 1 p, 1 p, 3 p+1 s$

$9 p+1 s+2 p$

Endopod

Exopod

Maxilla

Coxal endite

Basial endite

$$
9 \mathrm{p}+(1 \mathrm{~L}+2 \mathrm{e}+1 \mathrm{p})
$$

$$
(1 \mathrm{~L}+2 \mathrm{e}+1 \mathrm{p})+
$$$$
(1 \mathrm{~L}+2 \mathrm{e}+1 \mathrm{p})
$$

Endopod

$3 e+(1 h+1 e)+1 h+(1 h+$

$$
\text { 2e) }
$$

$5 \mathrm{p}$

Exopod

\section{Maxilliped}

$\begin{array}{lc}\text { Coxa } & 1 \mathrm{p}+1 \mathrm{p}+2 \mathrm{p} \\ \text { Basis } & 1 \mathrm{e}+2 \mathrm{p}+3 \mathrm{e}+3 \mathrm{e}+3 \mathrm{e} \\ \text { Endopod } & 3 \mathrm{e}, 1 \mathrm{e}, 2 \mathrm{e}, 1 \mathrm{~s}+3 \mathrm{r} \\ \text { Exopod } & 1 \mathrm{n}+3 \mathrm{n}\end{array}$

2 Maxilliped

Coxa

Basis

Endopod

Exopod
$1 \mathrm{e}$

$1 e+2 e+3 e+3 e$

$3 \mathrm{e}, 1 \mathrm{e}, 2 \mathrm{e}, 1 \mathrm{~s}+4 \mathrm{r}$

$2 n+3 n$
$2 \mathrm{p}+5 \mathrm{~d}$

$6 \mathrm{t}+1 \mathrm{~s}$

$(1 \mathrm{~s}+1 \mathrm{e}+1 \mathrm{~h}+)+(1 \mathrm{e}+1 \mathrm{~h})+$

$1 \mathrm{e}$

$1 \mathrm{p}$

$$
\begin{gathered}
10 p+(1 L+2 e+1 p) \\
(1 L+2 e+1 p)+ \\
(1 L+2 e+1 p)
\end{gathered}
$$

$3 e+(1 h+1 e)+1 h+(1 h+$

$$
\text { 2e) }
$$

$5 \mathrm{p}$

$$
\begin{gathered}
1 p+1 p+2 p \\
1 e+2 p+3 e+4 e+4 e \\
3 e, 1 e, 2 e, 1 s+3 r \\
1 n+4 n
\end{gathered}
$$

$1 \mathrm{e}$

$$
1 e+2 e+3 e+3 e
$$$$
3 \mathrm{e}, 1 \mathrm{e}, 0,2 \mathrm{e}, 1 \mathrm{~s}+4 \mathrm{r}
$$

$2 n+3 n$

$7 \mathrm{~d}$
$6 \mathrm{t}+1 \mathrm{~s}$
$(1 \mathrm{~s}+1 \mathrm{e}+1 \mathrm{~h}+)+(1 \mathrm{e}+$
$1 \mathrm{~h})+1 \mathrm{e}$

$1 \mathrm{p}$

$$
\begin{gathered}
11 p+(1 L+2 e+1 p) \\
(1 L+2 e+1 p)+ \\
(1 L+2 e+1 p)
\end{gathered}
$$

$3 e+(1 h+1 e)+1 h+(1 h+$

$$
\text { 2e) }
$$

$7 \mathrm{p}$

$$
\begin{gathered}
3 p+3 p+3 p \\
2 e+3 e+3 e+4 e+4 e \\
3 e, 1 e, 2 e, 1 s+3 r \\
1 n+4 n
\end{gathered}
$$

0

$$
\begin{gathered}
1 e+2 e+3 e+3 e \\
3 e, 1 e, 0,2 r, 1 s+4 r \\
2 n+4 n
\end{gathered}
$$

$$
7 d
$$

$6 \mathrm{t}+1 \mathrm{~s}$

$$
(1 \mathrm{~s}+1 \mathrm{e}+1 \mathrm{~h}+)+(1 \mathrm{e}+
$$$$
1 \mathrm{~h})+1 \mathrm{e}
$$

$1 \mathrm{p}$

$$
\begin{gathered}
11 p+(1 L+2 e+1 p) \\
(1 L+2 e+1 p)+ \\
(1 L+2 e+1 p) \\
3 e+(1 h+1 e)+1 h+(1 h+ \\
2 e) \\
7 p
\end{gathered}
$$

$$
\begin{gathered}
3 p+3 p+3 p \\
2 e+3 e+3 e+4 e+4 e \\
3 e, 1 e, 2 e, 1 s+3 r \\
1 n+4 n
\end{gathered}
$$

0

$1 e+2 e+3 e+3 e$

$3 e, 1 e, 0,2 r, 1 s+4 r$

$2 n+4 n$ 
3 Maxilliped

$\begin{array}{lcccc}\text { Coxa } & 0 & 0 & 0 & 0 \\ \text { Basis } & 1 \mathrm{e}+1 \mathrm{e}+1 \mathrm{e} & 1 \mathrm{e}+1 \mathrm{e}+1 \mathrm{e} & 1 \mathrm{e}+1 \mathrm{e}+1 \mathrm{e} & 1 \mathrm{e}+1 \mathrm{e}+1 \mathrm{e} \\ \text { Endopod } & 1 \mathrm{e}, 1 \mathrm{e}, 2 \mathrm{r}, 1 \mathrm{~s}+3 \mathrm{r} & 1 \mathrm{e}, 1 \mathrm{e}, 0,1 \mathrm{e}+2 \mathrm{r}, 1 \mathrm{~s}+3 \mathrm{r} & 1 \mathrm{e}, 1 \mathrm{e}, 1 \mathrm{e}, 1 \mathrm{e}+2 \mathrm{r}, 1 \mathrm{~s}+4 \mathrm{r} & 1 \mathrm{e}, 1 \mathrm{e}, 1 \mathrm{e}, 1 \mathrm{e}+2 \mathrm{r}, 1 \mathrm{~s}+4 \mathrm{r} \\ \text { Exopod } & 2 \mathrm{n}+3 \mathrm{n} & 2 \mathrm{n}+4 \mathrm{n} & 2 \mathrm{n}+2 \mathrm{n}+4 \mathrm{n} & 2 \mathrm{n}+2 \mathrm{n}+4 \mathrm{n}\end{array}$

1 Pereiopod

no

no

Biramous bud

Biramous bud

Coxa

Basis

Endopod

Exopod

2 Pereiopod

3 Pereiopod

Abdomen

3-3.5

3-3.5

Uropod

$\begin{array}{lcccc}\text { Protopod } & - & - & 0 & 0 \\ \text { Endopod } & - & - & 1 \mathrm{p} & 5 \mathrm{p} \\ \text { Exopod } & - & - & 6 \mathrm{p} & 6 \mathrm{p}\end{array}$

Telson

$(1 \mathrm{~L}+6 \mathrm{f})+(1 \mathrm{~L}+6 \mathrm{f})$

$(1 \mathrm{~L}+7 f)+(1 \mathrm{~L}+7 \mathrm{f})$

$(1 s+7 f)+(1 s+7 f)$

$(1 s+7 f)+(1 s+7 f)$

TABLE 1. (continued)

\begin{tabular}{cccc}
\hline Features & ZV & Z VI & Z VII \\
\hline
\end{tabular}

Duration of stage (days)

5

4

Cephalotorax

Length $(\mathrm{mm})$
Eyes
Ratio RS/FL

$1.117 \pm 0.028$

stalked funnel-shaped

$1.5-2$

Antennule

Peduncle
Endopod
Exopod

Antenna

Peduncle

Endopod

Ratio end/exo

Exopod

\section{Maxillule}

Coxal endite

Basial endite

Endopod

Exopod

Maxilla

Coxal endite

$11 \mathrm{p}+(2 \mathrm{e}+2 \mathrm{p})$

Basial endite

$1 c+5 p, 6 p, 4 p+7 p$

long plumose seta

$4 a+2 p$

$1 \mathrm{c}$

$1 \mathrm{~s}$

$>1 / 2$

$13 p+1 j+1 p$

$7 d$

$6 t+1 s$

$(1 \mathrm{~s}+2 \mathrm{e})+(2 \mathrm{r}+1 \mathrm{~d})$

$1 \mathrm{p}$
$2 p+1 c+6 p, 6 p, 5 p+5 p$

long plumose seta

$$
2 a+2 p
$$

$1 \mathrm{c}$

$1 \mathrm{~s}$

$3 / 4$

$13-15 p+1 j+1 p$

$1.135 \pm 0.029$

stalked funnel-shaped

$1.5-2$

.5-2

$3 p+1 c+10 p, 6 p, 7 p+5 p$

long plumose seta

$$
2 a+4 p
$$

$1 \mathrm{~s}+2 \mathrm{c}$

$0,1 \mathrm{~s}, 2 \mathrm{~s}+2 \mathrm{~s}$

1

$15 p+1 j+1 p$

$6 t+1 s$

$(1 \mathrm{~s}+2 \mathrm{e})+(2 \mathrm{r}+1 \mathrm{~d})$

0

$(1 \mathrm{r}+2 \mathrm{~d})+(2 \mathrm{r}+1 \mathrm{~d})$

0

$11-12 \mathrm{p}+(2 \mathrm{e}+2 \mathrm{p})$

$11 \mathrm{p}+(2 \mathrm{e}+2 \mathrm{p})$

$(1 \mathrm{~L}+2 \mathrm{e}+1 \mathrm{p})+(1 \mathrm{~L}+2 \mathrm{e}+1 \mathrm{p})$ 


$\begin{array}{lccc}\text { Endopod } & 3 \mathrm{e}+(1 \mathrm{~h}+1 \mathrm{e})+1 \mathrm{~h}+(1 \mathrm{~h}+2 \mathrm{e}) & 3 \mathrm{e}+(1 \mathrm{~h}+1 \mathrm{e})+1 \mathrm{~h}+(1 \mathrm{~h}+2 \mathrm{e}) & 3 \mathrm{e}+(1 \mathrm{~h}+1 \mathrm{e})+1 \mathrm{~h}+(1 \mathrm{~h}+2 \mathrm{e}) \\ \text { Exopod } & 9 \mathrm{p} & 9-10 \mathrm{p} & 12-13 \mathrm{p}\end{array}$

1 Maxilliped

$\begin{array}{lc}\text { Coxa } & 3 \mathrm{p}+3 \mathrm{p}+3 \mathrm{p} \\ \text { Basis } & 2 \mathrm{e}+3 \mathrm{e}+3 \mathrm{e}+4 \mathrm{e}+4 \mathrm{e} \\ \text { Endopod } & 3 \mathrm{e}, 1 \mathrm{e}, 2 \mathrm{r}, 1 \mathrm{~s}+3 \mathrm{r} \\ \text { Exopod } & 1 \mathrm{n}+4 \mathrm{n}\end{array}$

$1 p+1 p+(1 e+1 p)$
$2 e+(1 e+2 p)+(1 e+2 p)+4 e+4 e$
$3 e, 1 e, 2 r, 1 s+3 r$
$1 n+4 n$

$$
\begin{gathered}
1 p+1 p+(1 e+1 p) \\
2 e+(1 p+2 h)+(1 p+2 h)+4 e+4 e \\
3 e, 1 e, 2 r, 1 s+3 r \\
1 n+4 n
\end{gathered}
$$

2 Maxilliped

Coxa

0

0

$$
0
$$

Basis

Endopod

$1 e+2 e+3 e+3 e$

Exopod

$3 e, 1 e, 0,2 r, 1 s+4 r$

$2 n+2 n+4 n$

$1 s+2 e+3 e+3 e$

$3 \mathrm{e}, 1 \mathrm{e}, 0,2 \mathrm{r}, 1 \mathrm{~s}+4 \mathrm{r}$

$1 s+2 e+3 e+3 e$

$2 n+2 n+4 n$

$3 \mathrm{e}, 1 \mathrm{e}, 0,2 \mathrm{r}, 1 \mathrm{~s}+4-5 \mathrm{r}$

$2 n+2 n+4 n$

3 Maxilliped

Coxa

0

Basis

Endopod

$1 \mathrm{e}+1 \mathrm{e}+1 \mathrm{e}$

Exopod

$1 \mathrm{e}, 1 \mathrm{e}, 1 \mathrm{e}, 1 \mathrm{e}+2 \mathrm{r}, 1 \mathrm{~s}+4 \mathrm{r}$

$2 n+2 n+4 n$

0

$1 e+1 e+1 e$

$1 \mathrm{e}, 1 \mathrm{e}, 1 \mathrm{e}, 1 \mathrm{r}+3 \mathrm{r}, 1 \mathrm{~s}+4 \mathrm{r}$

$2 n+2 n+4 n$

0

$1 \mathrm{~s}+1 \mathrm{~s}+1 \mathrm{~s}$

1s, $2 \mathrm{~s}, 2 \mathrm{~s}, 2 \mathrm{r}, 1 \mathrm{~s}+3 \mathrm{r}$

$2 n+2 n+4 n$

Biramous bud

Biramous bud

2 Pereiopod

3 Pereiopod

Abdomen

Ratio $6^{\circ} / 5^{\circ}$

3-3.5

3-3.5

0

$2 \mathrm{p}+11 \mathrm{p}$

$4 \mathrm{p}+12 \mathrm{p}+1 \mathrm{~s}$

$4 \mathrm{p}+9 \mathrm{p}+1 \mathrm{~s}$

$(3 s+5 f)+(3 s+5 f)$

$(3 s+5 f)+(3 s+5 f)$

\section{0}

$1 \mathrm{e}+1 \mathrm{e}+1 \mathrm{e}$

1e, $1 \mathrm{e}, 1 \mathrm{e}, 1 \mathrm{r}+3 \mathrm{r}, 1 \mathrm{~s}+4 \mathrm{r}$

$2 n+2 n+2 n+4 n$

0

$1 \mathrm{~s}+1 \mathrm{~s}+1 \mathrm{~s}$

$2 \mathrm{~s}, 2 \mathrm{~s}, 1 \mathrm{~s}+1 \mathrm{r}, 3 \mathrm{r}, 1 \mathrm{~s}+3 \mathrm{r}$

$1 n+2 n+2 n+4 n$

i?

Biramous bud

3-3.5

0

$2 \mathrm{p}+13 \mathrm{p}$

\begin{tabular}{|c|c|c|c|c|c|c|c|}
\hline Instar & Eyes & $\begin{array}{c}\text { Antenna } \\
\text { segmentation }\end{array}$ & $\begin{array}{c}\text { Antenna ratio } \\
\text { end. / exop. }\end{array}$ & Uropod & $\begin{array}{c}\text { First } \\
\text { Pereiopod }\end{array}$ & $\begin{array}{c}\text { Second } \\
\text { Pereiopod }\end{array}$ & $\begin{array}{c}\text { Third } \\
\text { Periopod }\end{array}$ \\
\hline$Z I$ & sessile & yes & $1 / 2$ & - & - & - & - \\
\hline$Z I I$ & stalked & yes & $1 / 2$ & - & - & - & - \\
\hline ZIII & stalked & yes & $1 / 2$ & functional (end. bud) & bud & - & - \\
\hline$Z I V$ & stalked & no & $1 / 2$ & functional & bud & - & - \\
\hline$Z V$ & stalked & no & $>1 / 2$ & functional & functional & bud & - \\
\hline$Z V I$ & stalked & no & $3 / 4$ & functional & functional & bud & - \\
\hline ZVII & stalked & no & 1 & functional & functional & functional & bud \\
\hline
\end{tabular}

$6 \mathrm{p}+14 \mathrm{p}+1 \mathrm{~s}$

$(3 s+5 f)+(3 s+5 f)$

TABLE 2. Main features and time of appearance to reconnaissance the known larval stages of P. edwarsii. Abbreviations: end, endopod; exop, exopod; (-), absent. 
Third maxilliped (Figure 5G). Coxal endite without seta; basial endite with 3 sparsely plumose setae; endopod 4-segmented as long as exopod with 8 setae $(1,1,2,1+3)$. Exopod unsegmented with 2 subterminal and 3 terminal plumose natatory setae.
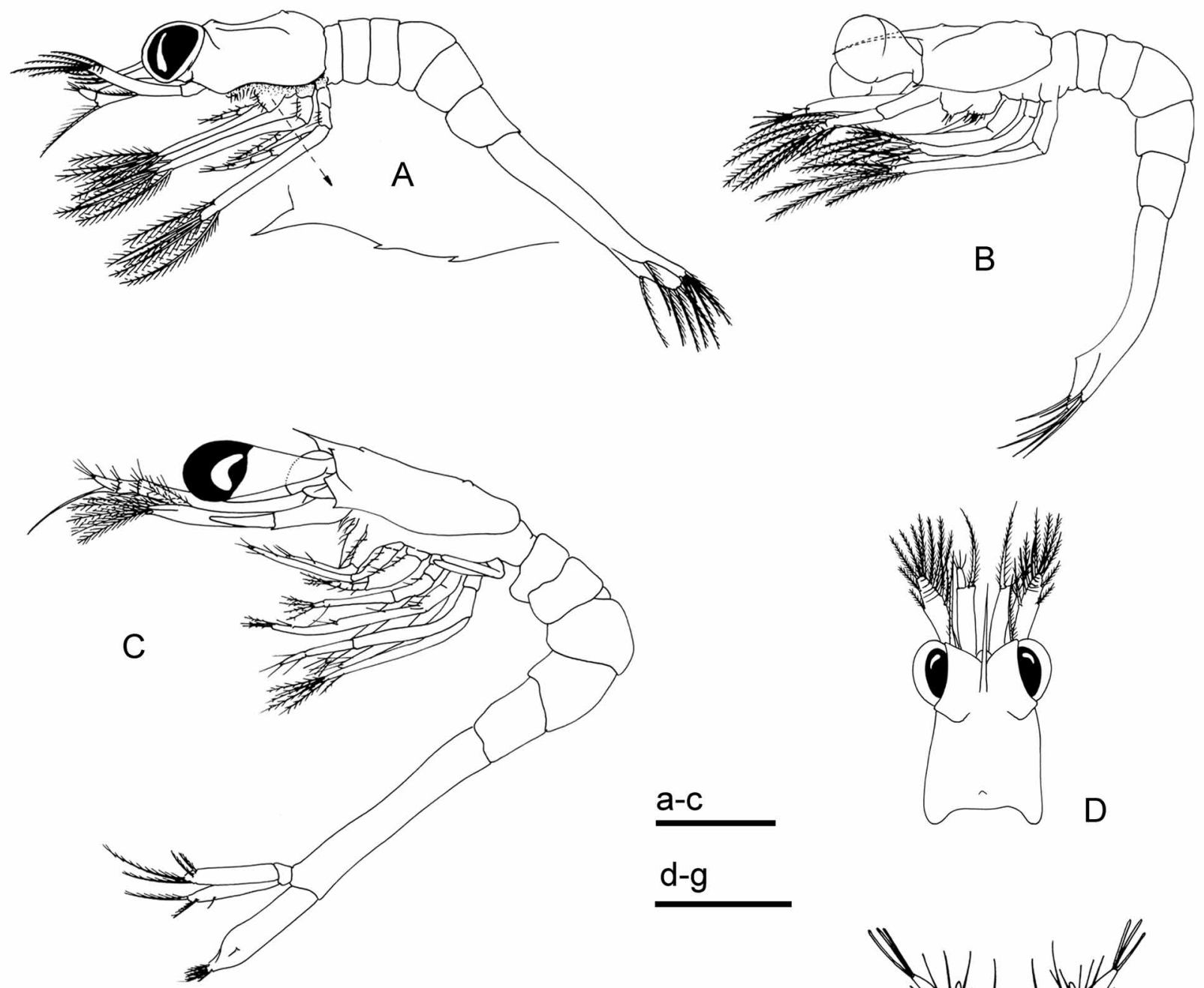

d-g
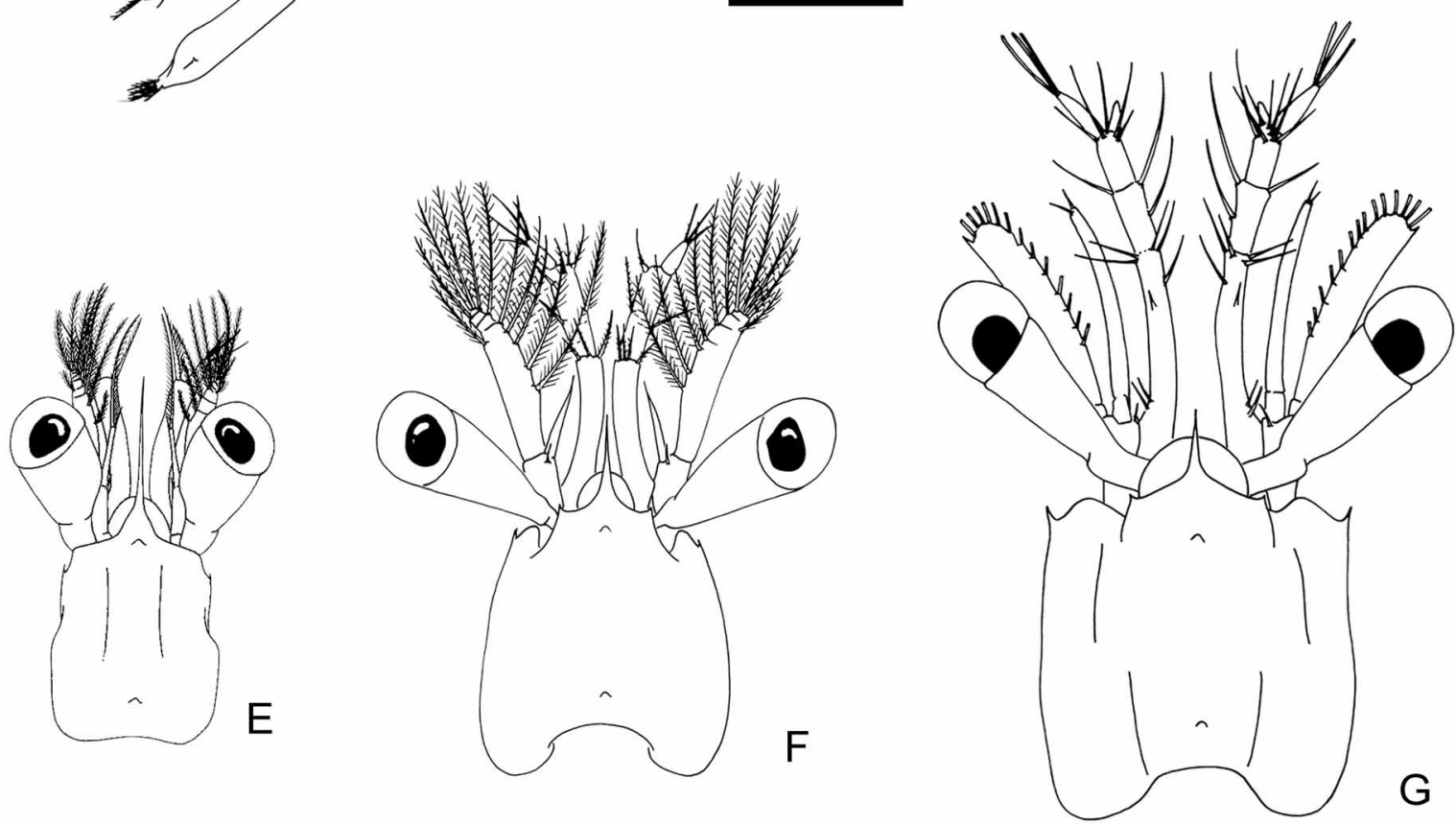

FIGURE 1. Plesionika edwardsii, lateral view: A, zoea I; B, zoea II; C, zoea VI; cephalotorax dorsal view: D, zoea I; E, zoea II; F, zoea III; G, zoea VII. Scale bars: $500 \mu \mathrm{m}$. 

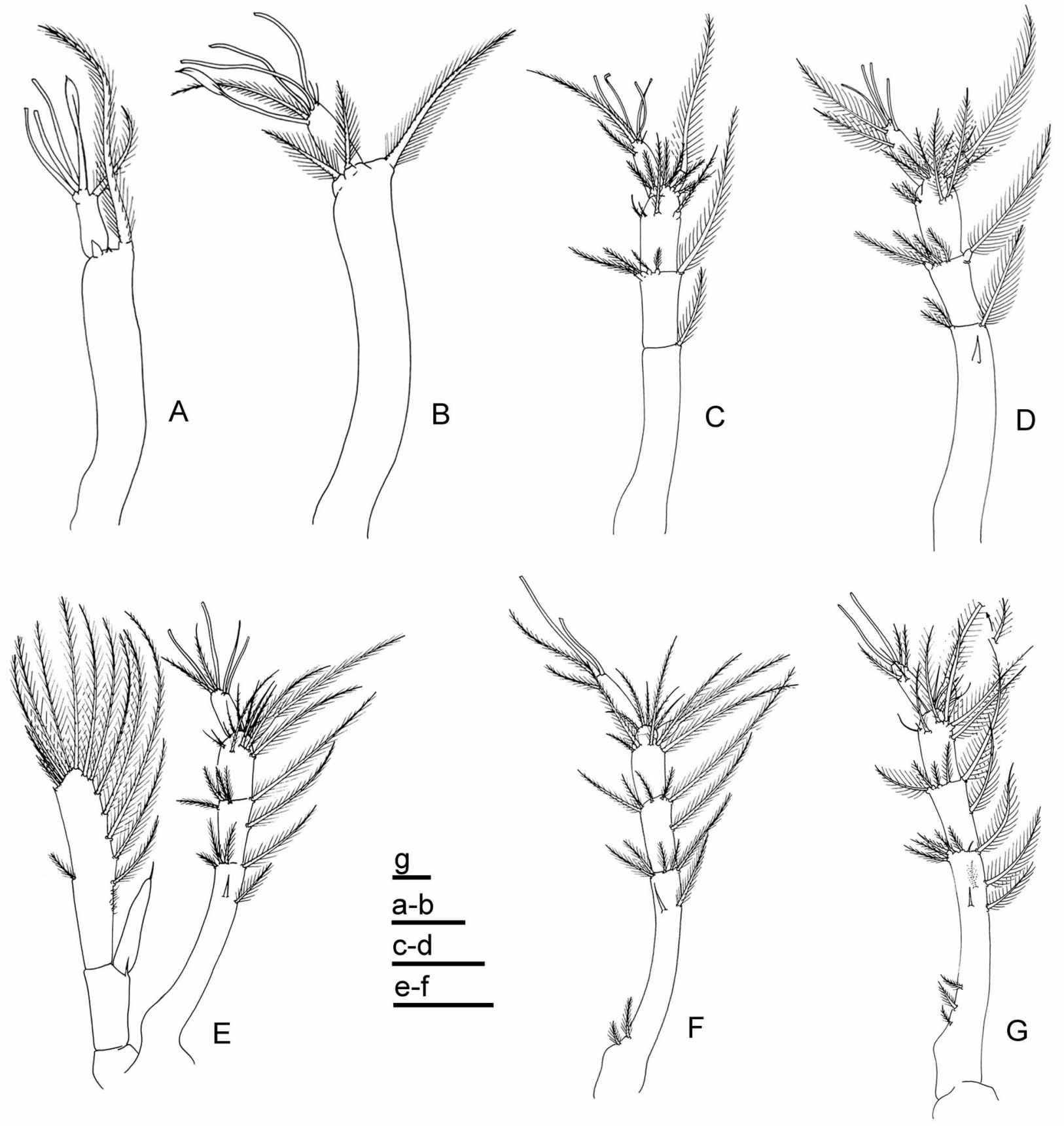

FIGURE 2. Plesionika edwardsii, antennule: A, zoea I; B, zoea II; C, zoea III; D, zoea IV; E, zoea V (and antenna); F, zoea VI; G, zoea VII. Scale bars: a-b, c-d, $g=100 \mu \mathrm{m}$; $-\mathrm{f}=250 \mu \mathrm{m}$.

Pereiopods. Absent.

Abdomen (Figure 1A). Five somites and telson. Anterior ventral margin slight flattened in somite 1. Somites without spines.

Pleopods. Absent.

Uropods. Absent.

Telson (Figure 7A). Triangular shape, broad in the posterior margin, with $7+7$ setae (inner 6 plumoserrulate, outer 1 laterally plumose setae). A row of spinules on distal margin and around base of setae. 

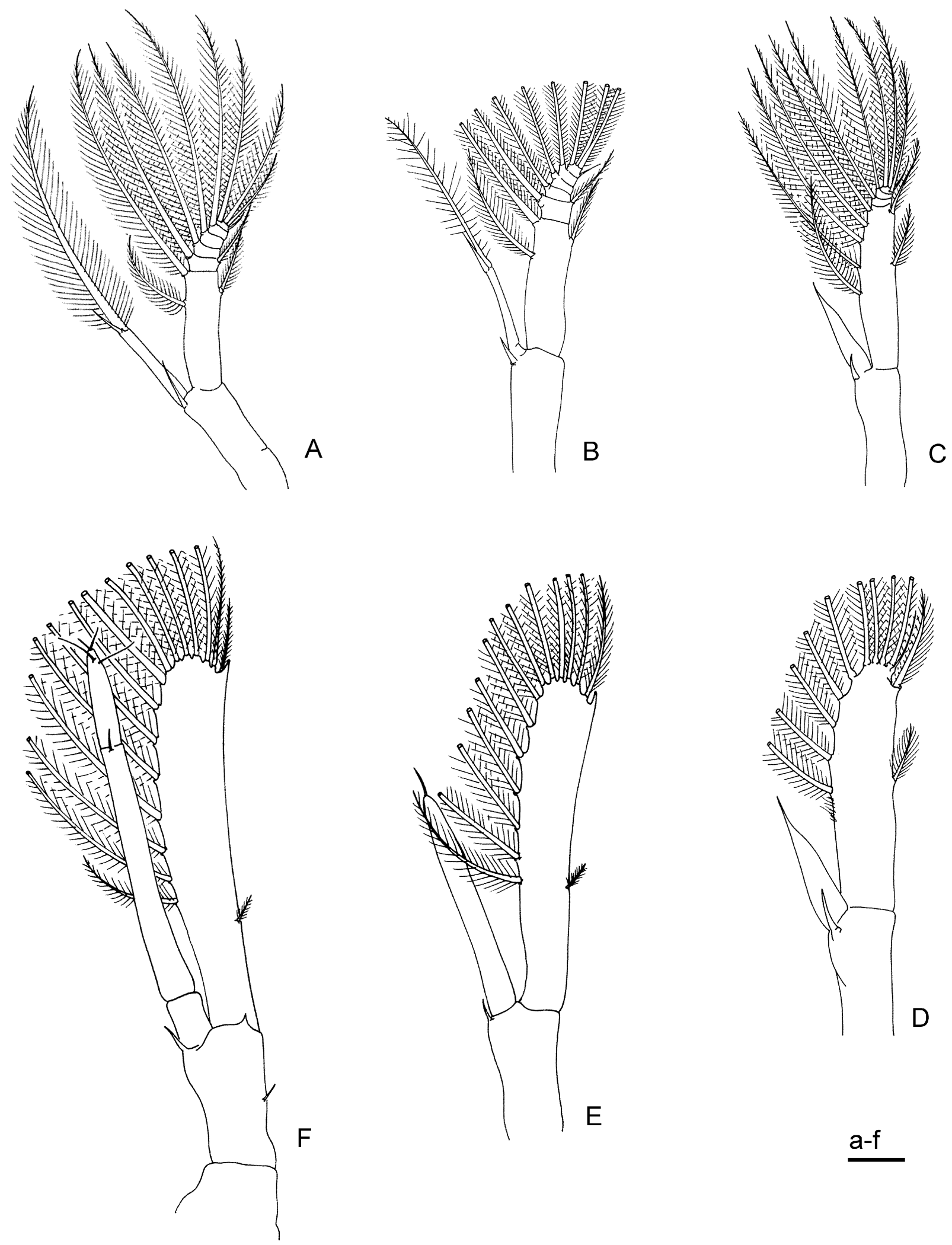

FIGURE 3. Plesionika edwardsii, antenna: A, zoea I; B, zoea II; C, zoea III; D, zoea IV; E, zoea VI; F, zoea VII. Scale bar: $100 \mu \mathrm{m}$.

\section{Following stages}

Cephalotorax (Figures 1E-G). Anterior ventral margin with a pterygostomial spine and two minute or absent anteroventral spines from zoea II. A pair of supraorbital spines appearing from zoea III on. 
Dorsomedian tubercle on anterior and posterior part of cephalotorax in all stages. Rostrum slender and pointed, reaching the extremity of the antennular peduncle in zoea II. The rostrum length is gradually reduced in the following stages but always longer than frontal lobe. Eyes stalked and funnel-shaped from zoea II on.
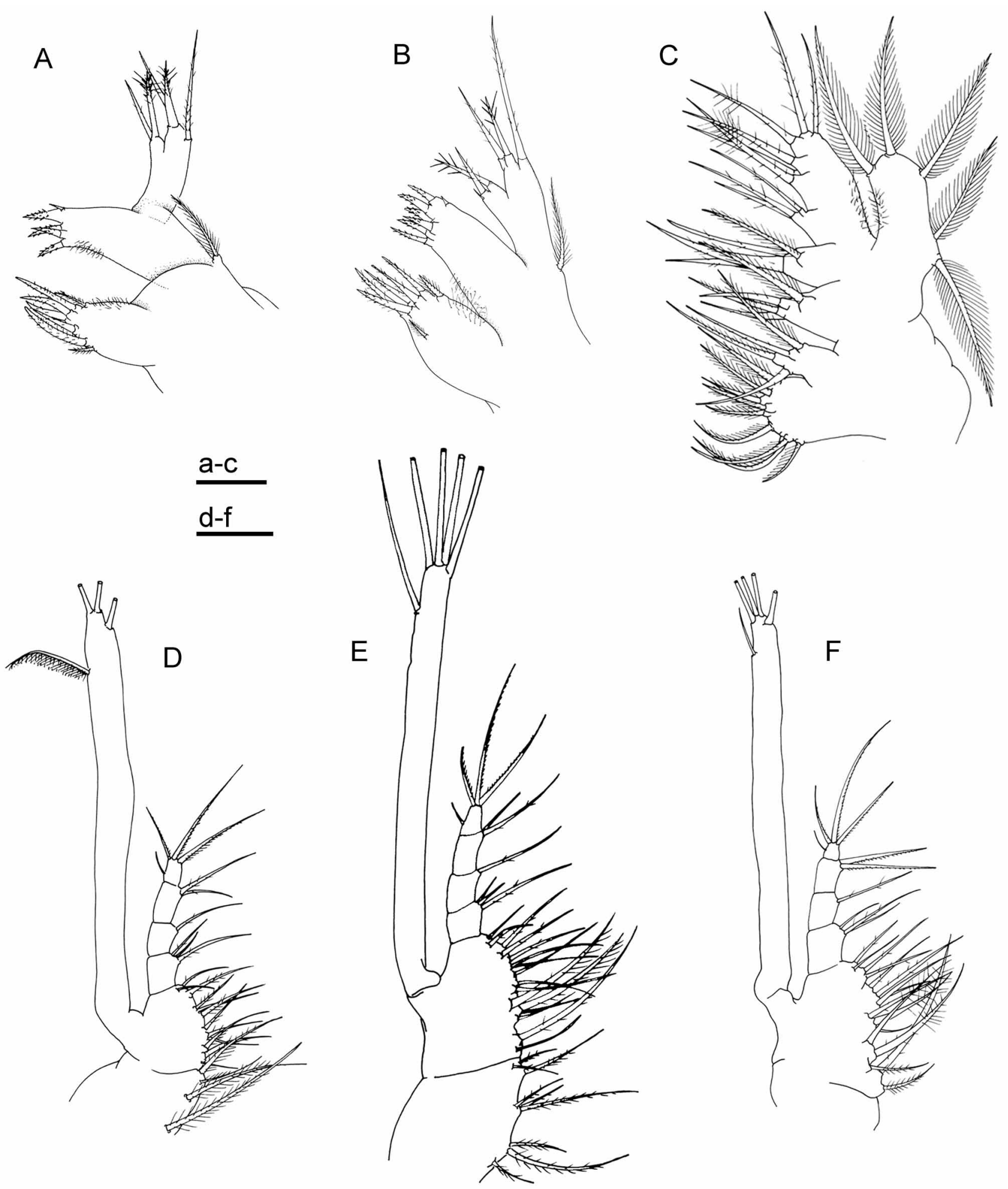

FIGURE 4. Plesionika edwardsii, maxillule: A, zoea I; B, zoea II; maxilla: C, zoea I; first maxilliped: D, zoea I; E, zoea III; F, zoea VII. Scale bars: $\mathrm{a}-\mathrm{c}=50 \mu \mathrm{m} ; \mathrm{d}-\mathrm{f}=100 \mu \mathrm{m}$. 

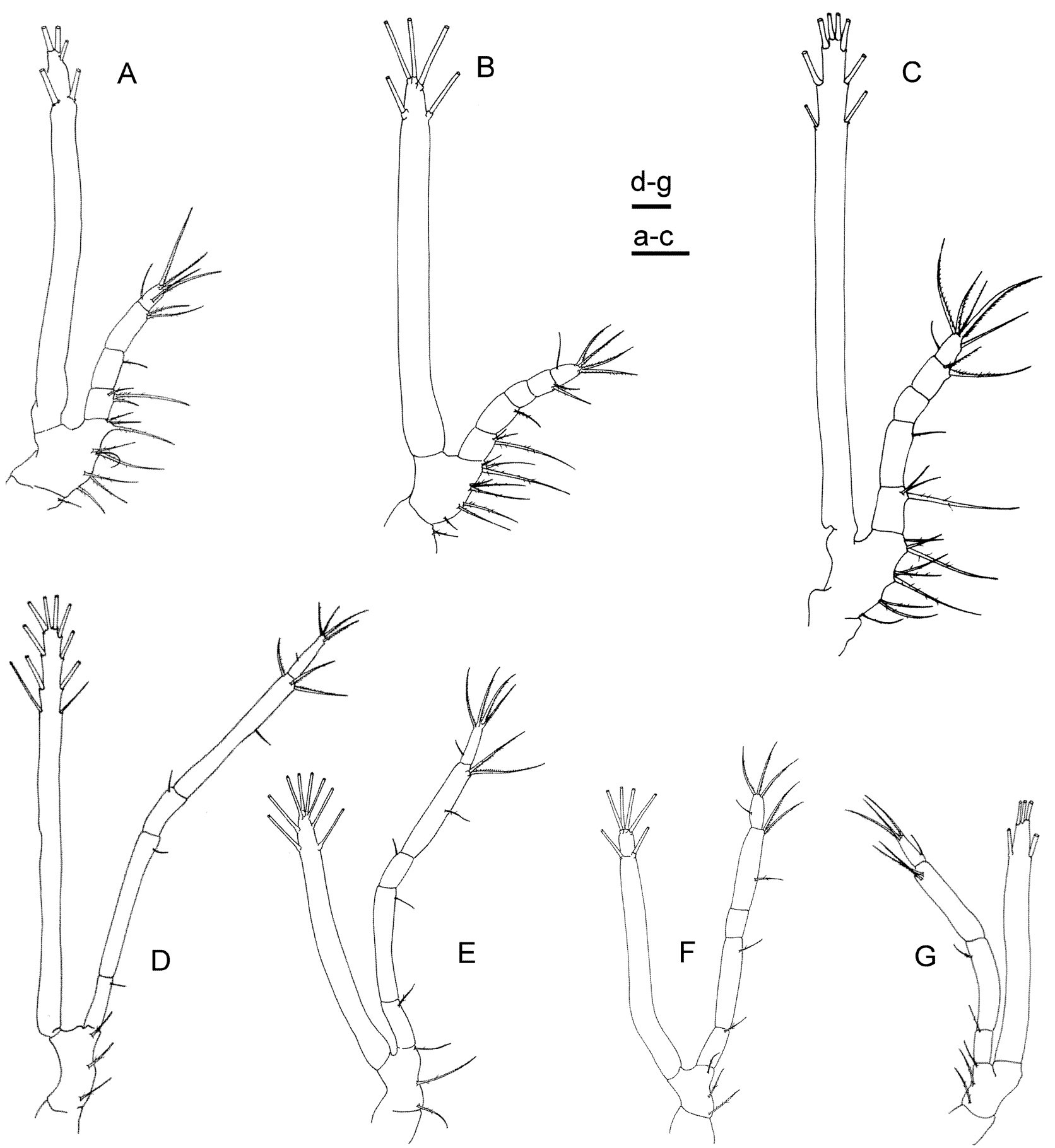

FIGURE 5. Plesionika edwardsii, second maxilliped: A, zoea I; B, zoea II; C, zoea VII; third maxilliped: D, zoea VII; E, zoea III; F, zoea II; G, zoea I. Scale bars: $100 \mu \mathrm{m}$..

Antennule (Figures 2B-E). Peduncle unsegmented in zoea II with 2 terminal plumose setae. Peduncle 3segmented from zoea III on, increasing the number of setae: zoea III (16 plumose setae), zoea IV (18 plumose and 1 spiniform setae), zoea V (22 plumose and 1 spiniform setae), zoea VI (24 plumose and 1 spiniform setae) and zoea VII (31 plumose and 1 spiniform setae); endopod as a long plumose seta; exopod with high setation variability, the spatulate seta disappears from zoea III.

Antenna (Figures 2E, 3B-F). Sharp spiniform seta in the peduncle, near the base of the endopod in all stages. Endopod unsegmented until zoea VII (when is 3-segmented), elongated with 2 terminal setae (long plumose and simple) until zoea II and extending beyond middle of exopod from the fifth stage to reach the same length in the zoea VII. Exopod broad and distally segmented only in the first three stages. 


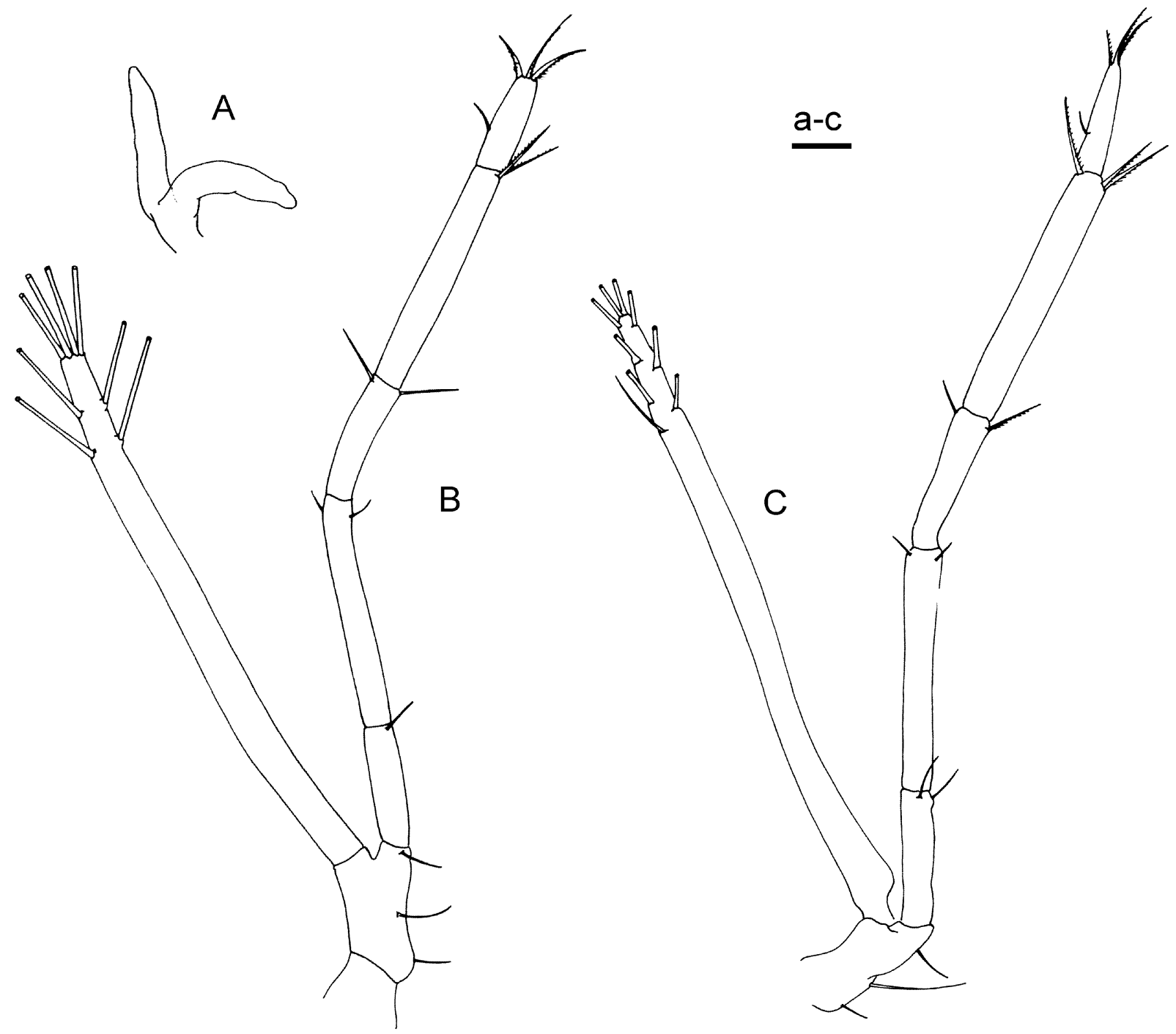

FIGURE 6. Plesionika edwardsii, first pereiopod: A, zoea III; B, zoea VI; C, zoea VII. Scale bar: $100 \mu \mathrm{m}$.

Mandible. Unchanged.

Maxillule (Figure 4B). Coxal endite with 7 setae until fifth stage and 8 setae in the following ones. Number of setae on basial endite and on endopod unchanged from zoea II on. Exopod presents as a long plumose seta until zoea $\mathrm{V}$.

Maxilla. Coxal endite bilobed, one of them with unchanged setation and other lobe increases number of plumose setae from 10 in zoea II to 11-12 in zoea VII. Basial endites and endopod with setation unchanged. Exopod increase the number of setae from 5 to 12-13 through development.

First maxilliped (Figures 4E, F). Endopod 4-segmented with unchanged setation $(3,1,2,1+3)$ and not extending beyond middle of exopod. Exopod unsegmented bearing 1 subterminal and 4 terminal plumose natatory setae.

Second maxilliped (Figures 5B, C). Coxa without seta from zoea III on. Basis with unchanged setation $(1+2+3+3)$. Endopod 5-segmented from zoea II with unchanged setation $(3,1,0,2,1+4)$ always extending beyond middle of exopod. Exopod unsegmented and the number of plumose natatory seta increase through development from $2+3$ setae in zoea II to $2+2+4$ setae in zoea VII.

Third maxilliped (Figures 5D, F). Coxa without seta. Basis unchanged. Endopod 4-segmented as long as exopod 5-segmented from zoea II on, exceeding length of exopod. Exopod unsegmented and number of plumose natatory seta increases through development from $2+4$ setae in zoea II to $2+2+2+4$ setae in zoea VII. 
First pereiopod. (Figures 6A, B, C). Biramous bud in third and fourth stages and fully functional from fifth stage on. Coxa without seta. Basis with unchanged setation $(1+1+1)$. Endopod longer than exopod, 4segmented in zoea $\mathrm{V}$ and 5-segmented in subsequent stages.

Second pereiopod. Biramous bud in fifth and sixth stages. Fully functional from seventh stage on. The segmentation and setation are not described because it was impossible to obtain undamaged appendages to analyse.

Third pereiopod. Biramous bud in zoea VII.

Abdomen (Figures 1B, C). Six abdominal somites from zoea III on. Anterior ventral margin slight flattened in somite I. Somites without setation.

Uropods (Figures 7C-E). Present from zoea III on.

Telson (Figures 7B-E). Triangular shape, broad in the posterior margin until zoea III and almost rectangular shaped in subsequent stages. A row of spinules on distal margin and around base of setae.
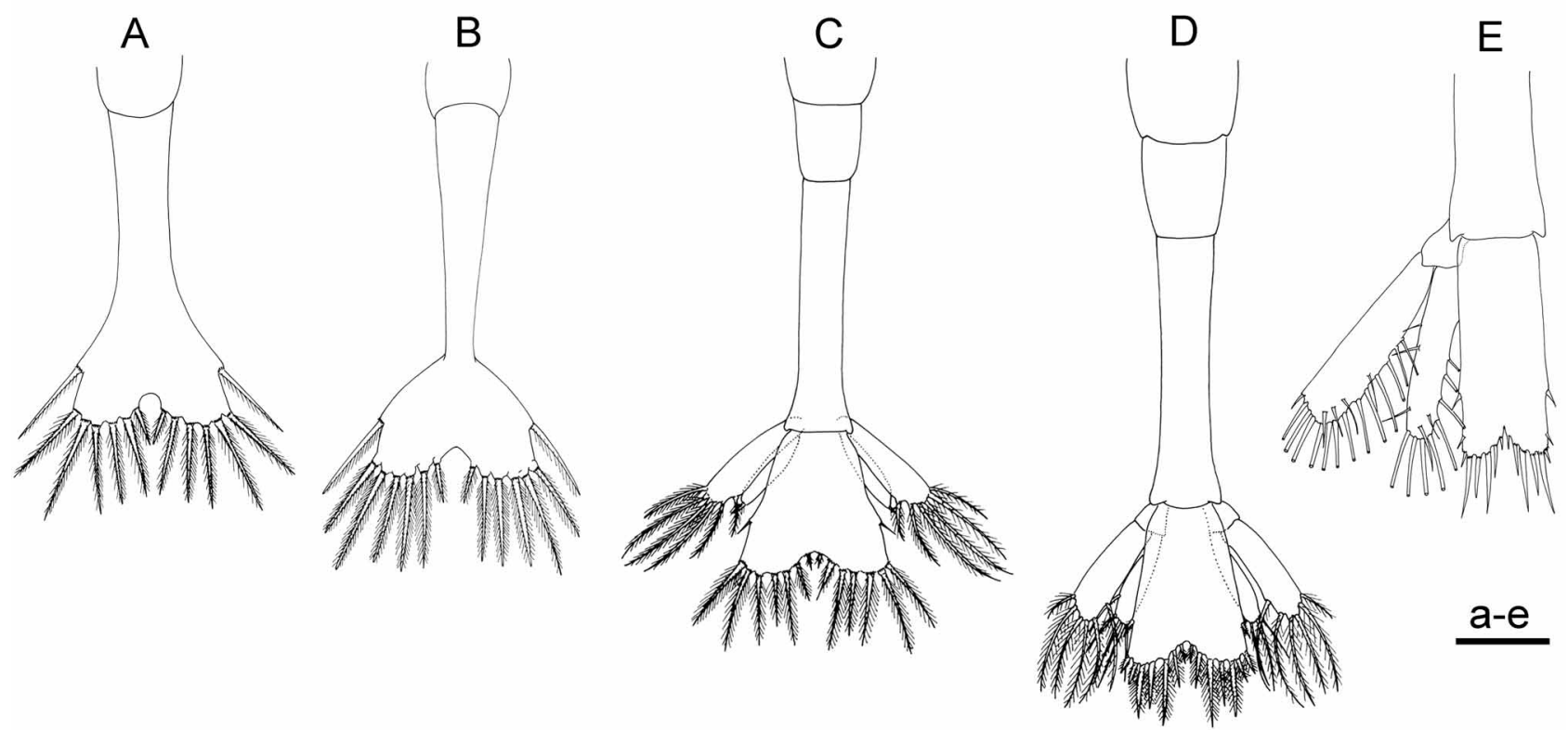

FIGURE 7. Plesionika edwardsii, telson: A, zoea I; B, zoea II; C, zoea III; D, zoea IV; E, zoea VI. Scale bar: $200 \mu$ m.

\section{Discussion}

Common morphological larval characters of Pandalidae have been documentented by Thatje \& Bacardit (2000) and summarized as follow: eye peduncle narrowed at base, antennular peduncles strongly concave, rostrum well developed since first stage, supraorbital spines present and cephalotorax with two dorsal protuberances.

Larvae of the family Pandalidae have been closely related to those of Hippolytidae and Processidae (Haynes 1985; Ortega et al. 2005) but strongly contrasted with the Alpheidae and Palaemonidae (Lebour 1940). Barnich (1996) found a character common to Hippolytidae and Pandalidae: the presence of a particular type of seta located on the antennular exopod (flattened apically and surrounded by a fine membrane). This spatulate shape seta has been specifically reported in the first zoeal stage of Hippolytidae (genus Caridion, Eualus, Lysmata, Saron and Thoralus) (Gurney 1937; Bourdillon-Casanova 1960; Le Roux 1963; Barnich 1996) and Pandalidae (genus Pandalina) (Barnich 1996). This particular setal type has been also observed in the first two larval stages of $P$. edwardsii. Nevertheless, as this seta has been reported only for two genera of the family Pandalidae (Pandalina and Plesionika), its presence in the remaining genera must be confirmed in order to evaluate its taxonomic importance. This characteristic morphological feature is likely to represent a clue to the phylogenetic relationships between these families. 
Notwithstanding, there are obvious dissimilarities between Hippolytidae and Pandalidae, namely the distance between the bases of antennules lower than the width of each antennule and the presence, in Hippolytidae larvae, of an anal spine the first larval stage onward. In Processidae, as in Pandalidae, the distance between bases of antennules is higher than the width of each antennule, but the presence of a segmented antennal exopod (early stages) and toothed-rostrum (late stages) are distinctive characters of pandalid larvae.

The zoeal morphology of Plesionika edwardsii presented herein is characterized by the combination of the following features: stalked and funnel-shaped eyes (from zoea II), rostrum longer than frontal lobe, bases of antennules separated by more than width of one of them, abdomen without lateral or dorsal spines, long sixth abdominal somite (3-3.5 longer than fifth one) and cephalotorax with two anteroventral spines in zoea I (minute or absent in the following stages).

Up to now, the Plesionika specimens from plankton samples collected in european waters have been sorted following the identification key proposed by Dos Santos \& González Gordillo (2004). However, the lack of knowledge on the larval morphology of genera Bitias Fransen, 1990, Chlorotocus Milne-Edwards, 1882, Heterocarpus Milne-Edwards, 1881 and Physetocaris Chace, 1940 (González-Gordillo et al. 2001), which have been also recorded from north-eastern Atlantic Ocean (Udekem D`Acoz 1999), raised our suspect that some larvae assigned to Plesionika can belong to any of these unknown genera.

Larval descriptions of the genus Plesionika from laboratory culture are rather unknown. At the moment, the only larval description available for a Plesionika species from laboratory culture was the one presented by Bourdillon-Casanova (1960) for the first zoeal stage of Plesionika acanthonotus. Both, P. acanthonotus and $P$. edwardsii species show morphological similarities in the first zoeal stage, such as the length of rostrum, distally located long spiny seta on the peduncle of the antenna, endopod of the antenna with a spiny seta and long plumose seta and absence of abdominal spines. On the other hand, we agree with Pike \& Williamson (1964) to differentiate the species (only the first zoeal stage) by the presence of denticles on the ventral cephalotorax margin in P. edwardsii. Also, the absence of seta on the peduncle of the antennule in P. edwardsii could be useful to differentiate both species.

Regarding the larvae found in plankton samples, Lebour (1940) suggests that the ones described as Icotopus by Bate (1888), Coutière (1907) and Gurney (1924) should belong to Plesionika. Despite the data provided in the present study, the identity of Icotopus remains unresolved, because these larvae appear to be more developed than the described specimens of $P$. edwardsii in this study. We think Icotopus represents a pandalid group that contains some genus with undescribed larval development. In addition, Williamson (1967a, 1967b) reported Plesionika martia? and Icotopus EM2 from last stage larvae which we were not able to confirm either.

On the other hand, other authors have reported larvae apparently more related to our specimens. Kurian (1956) presents a complete series of the larval stages of Plesionika sp.? from plankton tows in Dubrovnik region (eastern Mediterranean Sea). The first larval stage agrees with our description, despite some differences in the setation of antenna and antennule and the presence of denticles on the ventral cephalotorax margin. Other specimens described by Seridji (1971) from plankton samples collected in Alger, and assigned to the first two larval stages of Plesionika sp., show a denticulation pattern of the cephalotorax margin similar to the one described herein ( 2 denticles in first stage and 0 in the second one). However there are some differences between both larvae: Seridji's Plesionika larvae present less segmentation in the exopod of the antenna and one more aesthetasc in the exopod of the antennule.

Concerning the larvae assigned as Plesionika sp. by Barnich (1996), zoea I is identical to P. edwardsii, but some differences were found in the appearance of the first and second pereiopod bud in zoea II and zoea III, respectively, and in the presence of denticles on the rostrum from zoea IV on.

Finally, Dos Santos (1999) colleted Plesionika larvae every month for one year (excepting February and August), in plankton samples collected in the Portuguese coast. The author presented a description of a larval set (from zoea I to zoea VII) of Plesionika FSL12. The first two larval stages of Dos Santos (1999) do not differ from our larvae, but the presence of denticles on the rostrum and the persistence of denticles in the 
ventral cephalotorax allow distinguishing between both Plesionika larvae. In fact, the earlier appearance of the setated pleopods in Plesionika FSL12 suggests a shorter larval development. In this respect, the absence of the third pereiopod and pleopods in the abdomen of the zoea VII of Plesionika edwardsii suggest a longer larval series, probably up to ten or eleven stages. As a consequence of this longer larval development, Pike and Williamson (1964) considered Plesionika to be the most primitive genus of Pandalidae.

The high number of species assigned to the genus Plesionika makes it very difficult to relate our specimens with the larvae previously described from plankton samples. Our data suggests that these larvae must belong to other Plesionika species, or perhaps to another pandalid genus close to Plesionika.

Further studies must be conducted to increase the survival during culture. It is possible that the use of a getting darkness and cooling systems could improve the survival of the ovigerous females. In addition, to increase the larval survivorship, we think the use of bigger tanks could be more appropriated when the larvae moult to fifth zoeal stage because, they change their phototactic behaviour, preferring the bottom of the tank at this time. It is probably related to their natural ontogenetic migrations to their deep adult habitat.

\section{Acknowledgements}

The authors thank to José Ignacio Santana, José Antonio González and Ignacio José Lozano for providing the ovigerous females and to Daniel Montero and Javier Roo from Instituto Canario de Ciencias Marinas (ICCM) for culture support. This work was partially funded by project REDECA (CICYT, CTM2005-07712C03-01/MAR) and by the Formación de Personal Investigador (FPI) grant to J.M.L. (BES-2005-10960). JIG-G was supported by the Ramón y Cajal Research Program from Spanish Science and Technology Ministry and FEDER.

\section{References}

Barnich, R. (1996) The larvae of the Crustacea Decapoda (Excl. Brachyura) in the plankton of the French Mediterranean coast. $\mathrm{PhD}$ thesis, Göttingen, Cuvillier Verlag. 189 pp.

Bate, C.S. (1888) Report of the Crustacea Macrura collected by H.M.S. Callenger during the years 1873-76. Report of the scientific results or the Voyage of H.M.S. Challenger, Zoology, 24, XC+1-42.

Bourdillon Casanova, L. (1960) Le meroplancton du Golfe de Marseille: Les larves de Crustacés Décapodes. Recuéil des Travaux de la Station Marine d'Endoume, 30, 18, 1-286.

Calado, R., Narciso, L., Morais, S., Rhyneb, A.L. \& Lin, J. (2003) A rearing system for the culture of ornamental decapod crustacean larvae. Aquaculture, 218, 329-339.

Chan, T.Y. \& Yu, H.P. (2000) A new deep-sea shrimp of the genus Plesionika Bate, 1888 (Crustacea: Decapoda: Pandalidae) from Taiwan. National Taiwan Museum Special Publication, 10, 119-127.

Clark, P.F., Calazans, D.K. \& Pohle, G.W. (1998) Accuracy and standardization of brachyuran larval descriptions. Invertebrate Reproduction and Development, 33, 127-144.

Colloca, F. (2002) Life cycle of deep-water pandalid shrimp Plesionika edwardsii (Decapoda, Caridea) in the central Mediterranean Sea. Journal of Crustacean Research. 22, 775-783.

Coutière, H. (1907) Sur quelques formes larvaires enigmatiques d'Eucyphotes, provenant des collections de S. A. S le Prince de Monaco. Bulletin de l'Institut Oceanographique. Monaco, 104, 1-70.

Dos Santos, A. (1999) Larvas de crustáceos decápodes ao largo da costa portuguesa. PhD thesis, University of Lisbon, Portugal. 285 pp.

Dos Santos, A. \& González-Gordillo, J.I. (2004) Illustrated key for the identification of the Pleocyemata (Crustacea: Decapoda) zoeal stages, from the coastal region of south-western Europe. Journal of the Marine Biological Association of the United Kingdom, 84, 205-227.

Felgenhauer, B.E. (1992) Internal anatomy of the Decapoda: An overview. In: Harrison, F.W. \& Humes, A.G. (Eds), Microscopic anatomy of invertebrates: Decapod Crustacea, 3. Wiley-Liss, New York, pp. 7-44.

Garm, A. (2004) Revising the definition of the crustacean seta and setal classification systems based on examinations of the mouthpart setae of seven species of decapods. Zoological Journal of the Linnean Society, 142, $233-252$.

González, J.A., Carrillo, J., Santana, J.I., Martínez Baño, P. \& Vizuete, F. (1992) La pesquería de Quisquilla, Plesionika 
edwardsii (Brandt, 1851), con tren de nasas en el Levante español. Ensayos a pequeña escala en Canarias. Informes técnicos Scientia Marina, 170, 31pp.

González, J.A., Quiles, J.A., Tuset, V.M., García-Díaz, M.M. \& Santana J.I. (2001) Data on the family Pandalidae around the Canary Islands, with first record of Plesionika antigay (Caridea). Hydrobiologia, 449, 71-76.

González-Gordillo, J.I., dos Santos, A. \& Rodríguez, A. (2001) Checklist and annotated bibliography of decapod Crustacea larvae from the Southwestern European coast (Gibraltar Strait area). Scientia Marina, 65(4), 275-305.

Gurney, R. (1924) British Antarctic "Terra Nova" Expedition, 1910. Crustacea, part IX-Decapod larvae. Natural History Report, Zoology, 8(2), 37-202.

Gurney, R. (1937) Larvae of Decapod Crustacea. IV. Hippolytidae. Discovery Reports, 14, 351-404.

Haynes, E. (1985) Morphological development, identification, and biology of larvae of Pandalidae, Hippolytidae, and Crangonidae (Crustacea, Decapoda) of the northern north Pacific Ocean. Fishery Bulletin, 83(3), $253-288$.

Holthuis, L.B. (1980) FAO species catalogue. Shrimps and prawns of the world. An annotated catalogue of species of interest to fisheries. FAO Fisheries Circular, 125(1), 1-271.

Ingle, R.W. (1992) Larval stages of northeastern Atlantic crabs. An illustrated key. Natural History Museum Publications and Chapman \& Hall, London. pp. xxi+1-363, figs 1-2, 40.

Kurian, C.V. (1956) Larvae of decapod crustacea from the Adriatic Sea. Acta Adriatica, 6(3), 1-108.

Lebour, M.V. (1940) The larvae of the Pandalidae. Journal of Marine Biology Assiciation of the United Kingdom, 24, 239-252.

Le Roux, A. (1963) Contribution a l'etude du developpement larvaire d'Hippolyte inermis Leach (Crustace, Decapode, Macroure). Comptes Rendus Hebdomadaire des Seances de l'Academie des Sciences D. Paris, 256, 3499-3501.

Mura, M. \& Pessani, D. (1994) Descrizione del primo stadio larvale e notizie sul periodo riproducttivo di alcune specie di decapodi. Biologia Marina Mediterranea, 1, 391-392.

Ortega, A., Queiroga, H. \& González-Gordillo, J.I. (2005) Planktonic stages of Processa macrodactyla (Decapoda: Caridea: Processidae) reared in the laboratory. Journal of Marine Biology Association of the United Kingdom, 85, 1449-1460.

Pike, R.B. \& Williamson, D.I. (1964) The larvae of some species of Pandalidae (Decapoda). Crustaceana, 6, $265-284$.

Pohle, G. \& Telford, M. (1981) Morphology and classification of decapod crustacean larvae setae: a scanning electron microscope study of Dissodactylus crinitichelis Moreira, 1901 (Brachyura: Pinnotheridae). Bulletin of Marine Science, 31, 736-752.

Santana, J.I., González, J.A., Lozano, I.J. \& Tuset, V.M. (1997) Life history of Plesionika edwardsii (Crustacea, Decapoda, Pandalidae) around the Canary Islands, eastern central Atlantic. South African Journal of Marine Science, 18, 39-48.

Seridji, R. (1971) Contribution a l'etude des larves crustaces decapodes en baie d'Alger. Pelagos, 3, 1-105.

Thatje, S. \& Bacardit, R. (2000) Larval development of Austropandalus grayi (Cunningham, 1871) (Decapoda, Caridea, Pandalidae) from the southwestern Atlantic Ocean. Crustaceana, 73, 609-628.

Thessalou Legaki, M. (1992) Reproductive variability of Parapandalus narval (Crustacea: Decapoda) along a depth gradient. Estuarine and Coastal Shelf Science, 44,339-350.

Udekem D’Acoz, C.D’ (1999) Inventaire et distribution des crustacés décapodes de l'Atalantique nord-oriental, de la Méditerraée et des eaux continentales adjacentes au nord de $25^{\circ}$ N. Patrimoines naturels (M.N.H.N./S.P.N.), Paris, France, 40, $383 \mathrm{pp}$.

Vafidis, D., Politou, C., Carbonell, A. \& Company, J. (2005) A review of the biology and fisheries of the genus Plesionika Bate, 1888 (Decapada, Caridea, Pandalidae) in European waters. Crustaceana, 78, 335-352.

Williamson, D.I. (1967a) Crustacea Decapoda: Larvae. IV. Caridea, Families Pandalidae and Alpheidae. Fiches d'Identification du Zooplancton, 109, 1-5.

Williamson, D.I. (1967b) On a collection of planktonic Decapoda and Stomatopoda (Crustacea) from the mediterranean coast of Israel. Bulletin of Sea Fisheries Research Station of Haifa, 45, 32-64.

Zariquiey Álvarez, R. (1968) Crustáceos decápodos ibéricos. Investigación Pesquera, 32, 1-510. 